\title{
Distributed Midpoint Chain Scission in Ultrasonic Degradation of Polymers
}

\author{
G. Sivalingam, Nitin Agarwal, and Giridhar Madras \\ Dept. of Chemical Engineering, Indian Institute of Science, Bangalore-12, India
}

DOI 10.1002/aic.10185

Published online in Wiley InterScience (www.interscience.wiley.com).

\begin{abstract}
The ultrasonic degradation of poly(bisphenol A carbonate), poly(E-caprolactone), and poly(vinyl acetate) was investigated with various solvents such as benzene, monochlorobenzene, and dichlorobenzene. The time evolution of molecular weight was determined using gel permeation chromatography. A limiting molecular weight was observed for all the systems and was a function of solvent properties. The degradation rates increased with increase in viscosity and decrease in vapor pressure. The polydispersity reached a maximum before reaching a constant value at longer times. The experimental data indicate that the breakage of the polymer is around midpoint, with a distribution rather than an ideal midpoint scission. A continuous distribution model with a stoichiometric kernel based on Lorentzian probability distribution function was developed to satisfactorily model the experimental data. () 2004 American Institute of Chemical Engineers AIChE J, 50: 2258-2265, 2004

Keywords: ultrasonic degradation, limiting molecular weight, midpoint chain scission, poly(bisphenol A carbonate), poly(e-caprolactone), poly(vinyl acetate)
\end{abstract}

\section{Introduction}

The ultrasonic-assisted processes for polymers have received increased attention because of the wider availability of sonochemical apparatus and the advent of advanced equipment for macromolecular characterization (Price, 1992). Ultrasonic degradation of polymers was carried out as early as the late $1930 \mathrm{~s}$ and discussed in the mid-1950s by Jellinek (1955). Since then, ultrasonic degradation has been investigated for various polymers such as polystyrene (Price and Smith, 1993a,b), polyvinyl acetate (Madras and Chattopadhyay, 2003a,b; Madras et al., 2000), poly(methyl methacrylate) (Price et al., 1992), dextran (Koda et al., 1993), and various other polymers, as summarized in a literature review (Price, 1990). Ultrasonic degradation, unlike chemical or thermal fragmentation, is a nonrandom process with fragmentation occurring roughly at the center of the molecule. Larger molecules degrade faster than smaller molecules and no degradation occurs below a certain limiting

Correspondence concerning this article should be addressed to G. Madras at Giridhar@chemeng.iisc.ernet.in.

(C) 2004 American Institute of Chemical Engineers molecular weight. The mechanism by which degradation occurs is attributed to the hydrodynamic forces that may originate as a result of increased frictional forces between the ultrasonically accelerated faster moving solvent molecules and the larger, less mobile macromolecules or the hydrodynamic forces resulting from the high pressure associated with the collapse of cavitation bubbles (Mason and Peters, 2002; Price et al., 1994). It has been hypothesized that when ultrasound propagates in solution, cavitations occur followed by the growth and rapid collapse of microbubbles (Price, 1990). Polymer chains near the collapsing microbubbles are caught in a high-gradient shear field, causing the polymer segments in this shear field to move at a higher velocity than the chains farther away from the collapsing cavity. This relative motion of the polymer segments and solvent produces induced stresses to the polymer chain that cause scission (Madras and McCoy, 2001; Price, 1990). Because the polymer undergoes scission under mechanical shearing, there is a significant propensity for the midpoint rather than random scission.

In ultrasound degradation, molecular weight decreases exponentially and reaches a limiting molecular weight (Koda et al., 1993; Price and Smith, 1993a). The effects of various 
parameters such as temperature, ultrasound intensity, dissolved gases, and concentrations of polymers have been investigated (Price and Smith, 1993a,b; Price et al., 1994). The increase in polymer concentration and dissolved gases reduces the degradation rates and increases the limiting molecular weight. The effects of solvents and oxidizing agents on the ultrasonic degradation of polymers have been reported (Madras and Chattopadhyay, 2003a,b). Many of these investigations are based on the time variation of the number-average molecular weight $\left(M_{n}\right)$ and do not report the temporal dynamics of the molecular weight distribution (MWD). The time evolution of polydispersity is necessary in understanding the mechanism of degradation (Koda et al., 1993). The variation of the number-average molecular weight with time is independent of mode of scission (McCoy and Madras, 1998). However, little information has been published on polydispersity (PD) and details of the degradation process are still obscure (Koda et al., 1993).

The study of kinetics of ultrasonic degradation is crucial to an understanding of the underlying mechanisms of degradation (Madras and McCoy, 2001). The modeling strategy for the determination of kinetic coefficients of ultrasonic degradation of polymers can be broadly divided into three approaches.

In the first approach, the degradation is followed with the number of bonds broken, determined through the change in the number-average molecular weight (Jellinek and White, 1951; Mostafa, 1956; Ovenall et al., 1958; Schmid, 1940).

The second approach uses models (Glynn et al., 1972; van der Hoff and Glynn, 1974) based on probability arguments, by use of a term called degradation index, using number-average molecular weights as a probability factor. Both approaches suffer from the main disadvantage that the degradation index was inadequate for the degradation of polymers with wide molecular weight distribution.

The third approach involves solution of population balances of the polymers and radicals formed during the degradation. This continuous distribution kinetics approach could give the fundamental information about the mode of scission with time dynamics of molecular weight and polydispersity (Madras and McCoy, 2001; Madras et al., 2000). The stoichiometric kernels used in these balances will determine the mode of scission. All the studies (Bak and Bak, 1959; Madras and Chattopadhyay, 2001a,b; Madras and McCoy, 2001; Madras et al., 2000) assume ideal midpoint chain scission (Dirac delta function at half of the initial molecular weight). However, experimental data suggest that the ideal midpoint chain scission may not be a correct assumption and a distribution around the midpoint should be considered (Price, 1990). Thus in the present study, a model based on continuous distribution kinetics, considering a distribution in breakage around the midpoint chain, has been proposed. This study investigates the use of the Lorentzian distribution to account for the scission around the midpoint of the chain to model the experimental data.

The objective of the present investigation is twofold:

(1) To study the effect of solvent properties such as kinematic viscosity, vapor pressure on the degradation rates, polydispersity variation, and limiting molecular weight during the degradation of various polymers such as poly(bisphenol A carbonate), poly( $\varepsilon$-caprolactone), and poly(vinyl acetate).

(2) To develop a continuous distribution theory based on Lorentzian probability density function as stoichiometric kernel to explain the observed experimental behavior.

\section{Experimental}

\section{Materials}

Poly(bisphenol A carbonate) $\left(M_{n}, 44,000 ;\right.$ PD, 1.4$)$, poly $(\varepsilon-$ caprolactone) $\left(M_{n}, 80,000 ; \mathrm{PD}, 1.3\right.$; Sigma Aldrich), poly(vinyl acetate) $\left(M_{n}, 226,000 ; \mathrm{PD}, 1.3\right.$; prepared by bulk polymerization), $o$-dichlorobenzene (DCB), monochlorobenzene (MCB), benzene, and tetrahydrofuran (THF) were procured from S. D. Fine Chemicals Ltd. (Mumbai, India). All the solvents were double distilled and filtered before use.

\section{Degradation experiments}

Samples $\left(2 \mathrm{~kg} / \mathrm{m}^{3}\right)$ of polymer solutions were made with different solvents. A $20-\mathrm{mL}$ sample of each solution was charged to a $50-\mathrm{cm}^{3}$ glass beaker and the beaker was kept in a constant temperature bath $\left( \pm 1^{\circ} \mathrm{C}\right)$. The temperature in the reaction vessel was constant at $25^{\circ} \mathrm{C}$, identical to that of the constant temperature bath, and was verified by measuring the temperature at periodic intervals of $15 \mathrm{~min}$. The sonic horn (Vibronics, $25 \mathrm{kHz}$ and $180 \mathrm{~W}$ ) was dipped into the solution of the beaker and the gap between horn and beaker was closed to reduce any vaporization loss of the solvent. The ultrasonic horn is switched on after the solution attained the bath temperature. Each experiment was conducted for 8 to $10 \mathrm{~h}$ to determine the limiting molecular weight and polydispersity. Samples of 200 $\mu \mathrm{L}$ were collected at various time intervals for analysis by gel permeation chromatography (GPC). Several experiments were repeated in triplicate and the variation in the number-average molecular weight was less than $2 \%$.

\section{Molecular weight determination}

The MWD of the samples was determined by GPC (Waters, Milford, MA), which consisted of an isocratic pump, three size-exclusion columns, a differential refractrometer (Waters R401), and a data-acquisition system. THF was used as eluent at the flow rate of $1 \mathrm{~mL} / \mathrm{min}$. The columns [Styragel HR 4, HR 3 , and HR $0.5(300 \times 7.5 \mathrm{~mm})]$, packed with crosslinked polystyrene-divinylbenzene, were used in series at $50^{\circ} \mathrm{C}$. Samples were injected in a Rheodyne valve with a $50-\mu \mathrm{L}$ sample loop and the refractive index was continuously monitored using a differential refractive index detector and stored digitally. The chromatograph was converted to MWD using the universal calibration with polystyrene standards (Polymer Lab, Poole, UK). The MW of polystyrene was converted to MW of the individual polymers using the Mark-Houwink equation. The parameters in the relationship $[\eta]=K M^{\alpha}$ are $K_{\mathrm{PS}}=1.25 \times$ $10^{-5} \mathrm{dL} / \mathrm{g}, \alpha_{\mathrm{PS}}=0.717 ; K_{\mathrm{PC}}=1.95 \times 10^{-4}, \alpha_{\mathrm{PC}}=0.692$; $K_{\mathrm{PCL}}=1.09 \times 10^{-5} \mathrm{dL} / \mathrm{g}, \alpha_{\mathrm{PCL}}=0.60 ; K_{\mathrm{PVAC}}=1.5 \times 10^{-5}$ $\mathrm{dL} / \mathrm{g}, \alpha_{\text {PVAC }}=0.709$. Further details are provided elsewhere (Sivalingam and Madras, 2003).

\section{Theoretical Model}

We develop a model for ultrasonic degradation of polymers using continuous distribution kinetics. The model is largely based on that developed by Madras and McCoy (2001) but accounts for scission around the midpoint. The polymer, $P(x)$, is considered to be a mixture of homologous molecules with molecular weight $x$, and undergoes binary fragmentation to the polymers of molecular weight $x^{\prime}$ and $x-x^{\prime}$ 


$$
P(x) \stackrel{k_{d}(x)}{\longrightarrow} P\left(x^{\prime}\right)+P\left(x-x^{\prime}\right)
$$

where $k_{d}(x)$ represents the rate coefficient for the binary breakage.

The distribution function is defined such that $p(x, t) d x$ defines the molar concentration of the polymer in the interval $[x$, $x+d x]$. The governing population balance equation describes the fragmentation of the polymer (Aris and Gavalas, 1966; Ziff, 1991), as follows

$$
\frac{\partial p(x, t)}{\partial t}=-\kappa p(x, t)+2 \int_{x}^{\infty} \kappa p\left(x^{\prime}\right) \Omega\left(x, x^{\prime}\right) d x^{\prime}
$$

The rate coefficient usually depends on molecular weight of the reacting polymers (McCoy and Madras, 1997). The rate constant $(\kappa)$ is a function of the molecular weight of the polymer and is given as

$$
\kappa=k_{d 0}\left(x-x_{\lim }\right)^{\lambda}
$$

In Eq. 2, $\lambda$ is the power law index accounting for the dependency of the rate coefficient on the molecular weight and $\lambda=$ 0 represents the independence of $\kappa$ on the molecular weight of polymer. The stoichiometric kernel should satisfy the symmetry and normalization conditions. $x_{\text {lim }}$ is the limiting molecular weight below which polymers do not undergo any scission that is a characteristic of ultrasonic degradation (Koda et al., 1993; Price and Smith, 1993a,b). The domain of $x$ is from $\left[x_{\text {lim }}, \infty\right)$. $\Omega\left(x, x^{\prime}\right)$ is the stoichiometric kernel that describes the probability of formation of polymer of size $x$ from breakage of polymer of size $x^{\prime}\left(x^{\prime}>x\right)$. Several types of stoichiometric kernels are available (Hill and Ng, 1995; McCoy and Wang, 1994). In the case of ultrasonic degradation, the stoichiometric kernel is written in the form of a Dirac delta function, $\delta(x-$ $x^{\prime} / 2$ ) (Madras and Chattopadhyay, 2001a,b; Madras and McCoy, 2001; Madras et al., 2000). In the present study, the stoichiometric kernel is taken as a Lorentzian function, which allows a distribution around the midpoint of polymer based on its single parameter, instead of a Dirac delta function

$$
\Omega\left(x, x^{\prime}\right)=\frac{1}{I}\left[\frac{1}{\left(x-x^{\prime} / 2\right)^{2}+(\Gamma / 2)^{2}}\right]
$$

In Eq. 3, I $=\Gamma /\left[4 \tan ^{-1}(x / \Gamma)\right]$ and satisfies normality. The function reduces to a Dirac delta function at $x=x^{\prime} / 2$ and $\Gamma=$ 0 .

\section{Modifications in the PBE}

Equation 1 is solved with Eqs. 2 and 3 using the following dimensionless variables (Madras and McCoy, 2001)

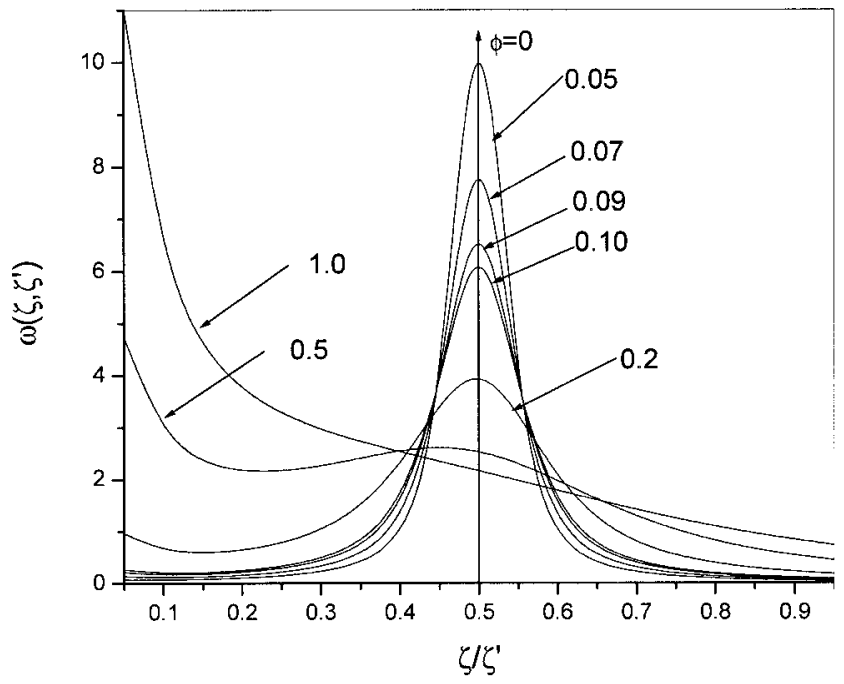

Figure 1. Variation of the stoichiometric kernel $\omega\left(\zeta, \zeta^{\prime}\right)$ (Eq. 6) with $\zeta / \zeta^{\prime}$ for various values of $\phi$ with $\zeta^{\prime}$ $=1$.

$$
\begin{aligned}
& p(\zeta, \theta) d \theta=p(x, t) d x / p_{0}^{(0)} \\
& p(\zeta, \theta)=p(x, t) M_{n 0} / p_{0}^{(0)} \\
& \zeta=x / M_{n 0} \\
& \omega\left(\zeta, \zeta^{\prime}\right)=\Omega\left(x, x^{\prime}\right) M_{n 0} \\
& \kappa(\zeta)=k_{d}(x) / k_{d 0} \\
& \theta=t k_{d 0}
\end{aligned}
$$

where $M_{n 0}$ is the initial molecular weight of the polymer and $p_{0}^{(0)}$ is the initial zeroth moment. Equation 1 becomes

$$
\begin{aligned}
& \frac{\partial P(\zeta, \theta)}{\partial \theta}=-\left(\zeta-\zeta_{\lim }\right)^{\lambda} p(\zeta, \theta) \\
& \quad+2 \int_{\zeta}^{\infty} \omega\left(\zeta, \zeta^{\prime}\right)\left(\zeta^{\prime}-\zeta_{\lim }\right)^{\lambda} p\left(\zeta^{\prime}, \theta\right) d \zeta^{\prime}
\end{aligned}
$$

The stoichiometric kernel (Eq. 3) takes the following form

$$
\omega\left(\zeta, \zeta^{\prime}\right)=\frac{\phi}{4 \tan ^{-1}(\zeta / \phi)}\left[\frac{1}{\left(\zeta-\zeta^{\prime} / 2\right)^{2}+(\phi / 2)^{2}}\right]
$$

The parameter $\phi\left(=\Gamma / M_{n 0}\right)$ accounts for the mode of breakage from close to midpoint scission $(\phi \ll 1)$ to random degradation $(\phi \gg 1)$. Figure 1 shows the variation of $\omega\left(\zeta, \zeta^{\prime}\right)$ as a function of $\zeta / \zeta^{\prime}$ for various values of $\phi$ with $\zeta^{\prime}$ of unity. Because there is no tension at the end of the polymer during fragmentation, the scission probability should go to zero as the ends are reached. Given that the fragmentation occurs preferentially at the center, the distribution should be peaked at the center. For the values of $\phi$ ( 0 to 0.1$)$ chosen in this study, the distribution is peaked at the center $\left(\zeta / \zeta^{\prime}=0.5\right)$ and the ends of the distribution are cut off at zero, as desired. Another advantage of choosing a Lorentzian distribution as a stoichiometric kernel is that a wide range of scissions (midpoint to random) can be represented by choosing the appropriate value of $\phi$. The 


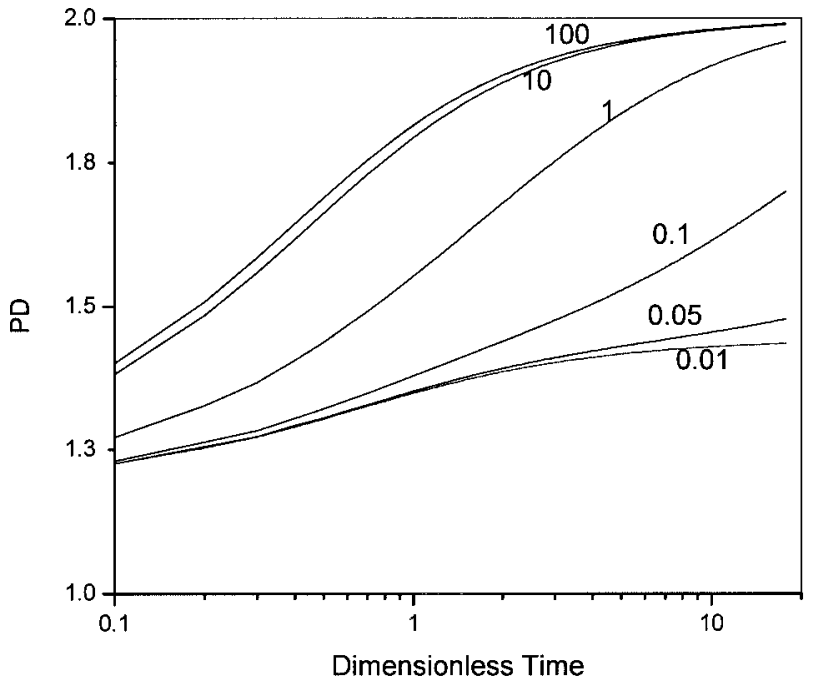

(a)

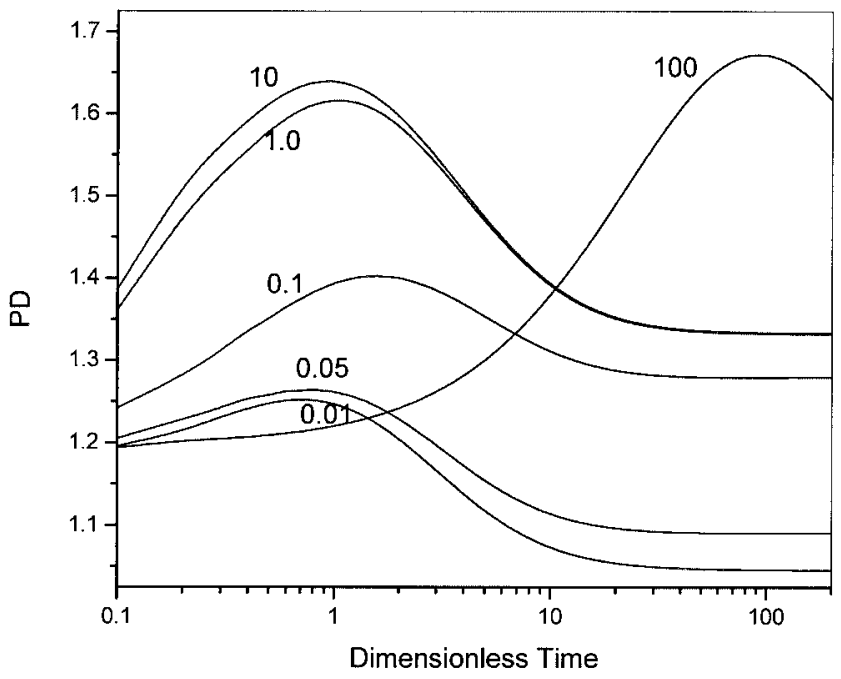

(c)

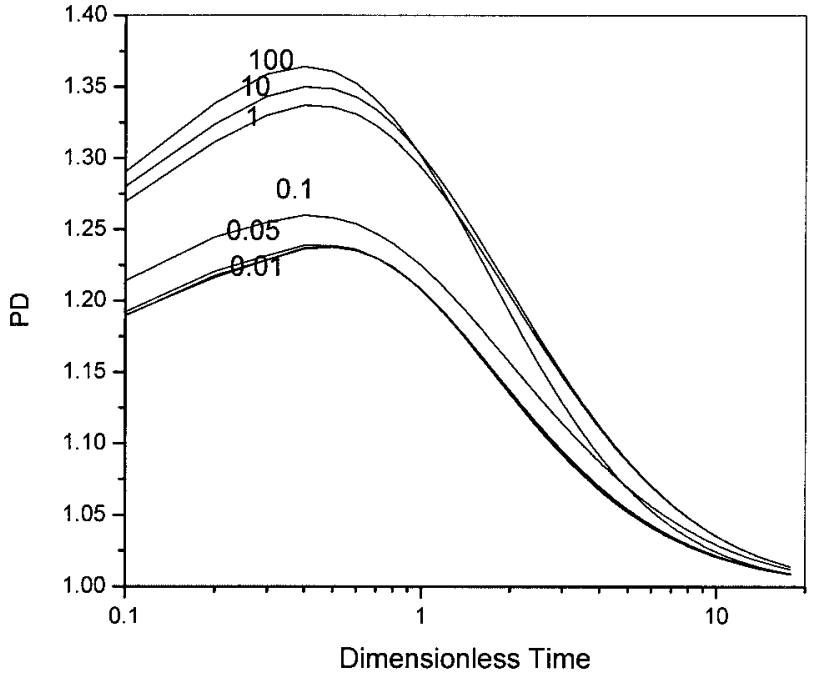

(b)

Figure 2. Theoretical time evolution of polydispersity (PD) for different values of $\phi$.

$M_{n 0}=4.0, \mathrm{PD}=1.15$ for (a) $\zeta_{\lim }=0.0$ with definition of moment by Eq. 7 ; (b) $\zeta_{\lim }=0.5$ with definition of moment by Eq. 7 ; (c) $\zeta_{\lim }=0.5$ with definition of moment by Eq. 8 .

rate coefficient is taken to be a linear function of molecular weight so that $\lambda=1$. The PBE (Eq. 5) is solved numerically for different values of $\zeta_{\lim }(=0$ to 1.0$)$ for a Lorentzian probability distribution (Eq. 6) and $\phi=0.01-100$ with given initial distribution. The moments for a distribution $p(\zeta, \theta)$ can be defined as (Madras and McCoy, 2001)

$$
p^{(j)}(\theta)=\int_{\zeta \lim }^{\infty} \zeta^{j} p(\zeta, \theta) d \theta
$$

or as

$$
p^{(j)}(\theta)=\int_{0}^{\infty} \zeta^{j} p(\zeta, \theta) d \theta
$$

Polymers with MW lower than $\zeta_{\text {lim }}$ cannot degrade in ultrasonic degradation. However, degradation can lead to the poly- mers lower than $\zeta_{\text {lim }}$. For example, polymers with MW between $\zeta_{\text {lim }}$ and $2 \zeta_{\text {lim }}$, undergoing midpoint chain scission, will lead to polymers of MW $<\zeta_{\text {lim }}$. Thus Eq. 8 is more appropriate for the calculation of moments. The temporal dynamics of the molecular weight and polydispersity are calculated using the following expressions $M_{n}=p^{(1)} / p^{(0)}$ and $M_{w}=p^{(2)} / p^{(1)}$ and the polydispersity $D=M_{w} / M_{n}$.

\section{Results and Discussion Model validation and simulations}

The governing PBE was solved with $\lambda=1$ numerically using the Lorentzian probability distribution as stoichiometric kernel for different values of $\zeta_{\text {lim }}$ and the function parameter $\phi$. Figures $2 \mathrm{a}$ and $\mathrm{b}$ show the time evolution of polydispersity for various values of $\phi$ for $\zeta_{\text {lim }}=0$ and 0.5 , respectively. The initial distribution was assumed to be a gamma distribution with the average molecular weight $\left(M_{n 0}\right)$ and polydispersity (PD) in nondimensional form of 4.0 and 1.15, respectively (Madras and McCoy, 2001). It is observed 


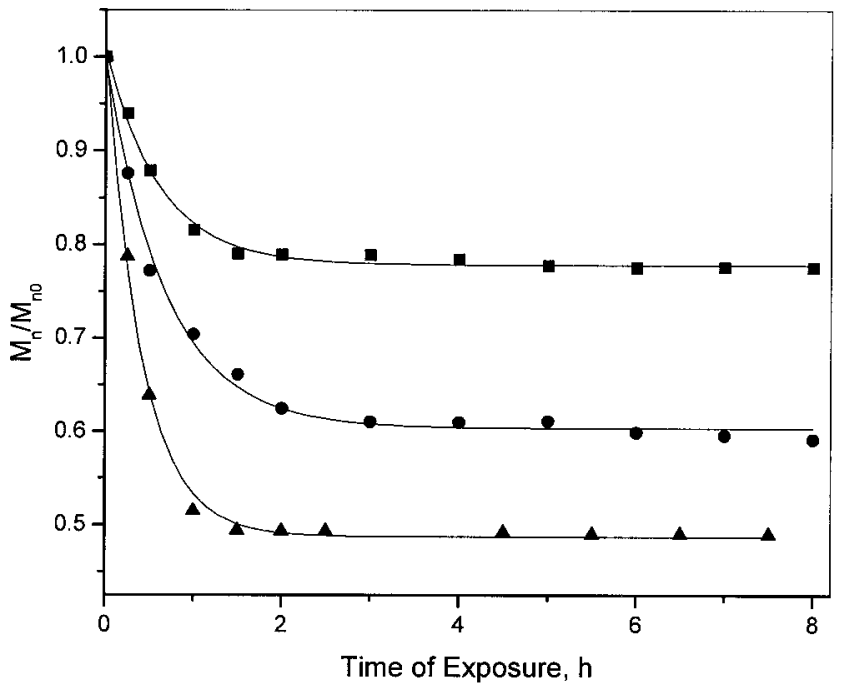

(a)

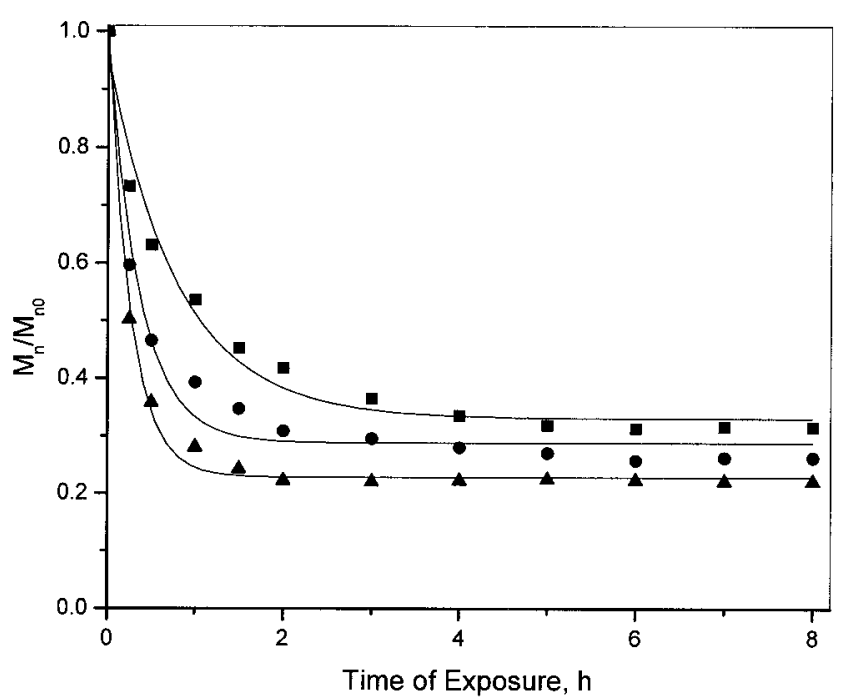

(c)

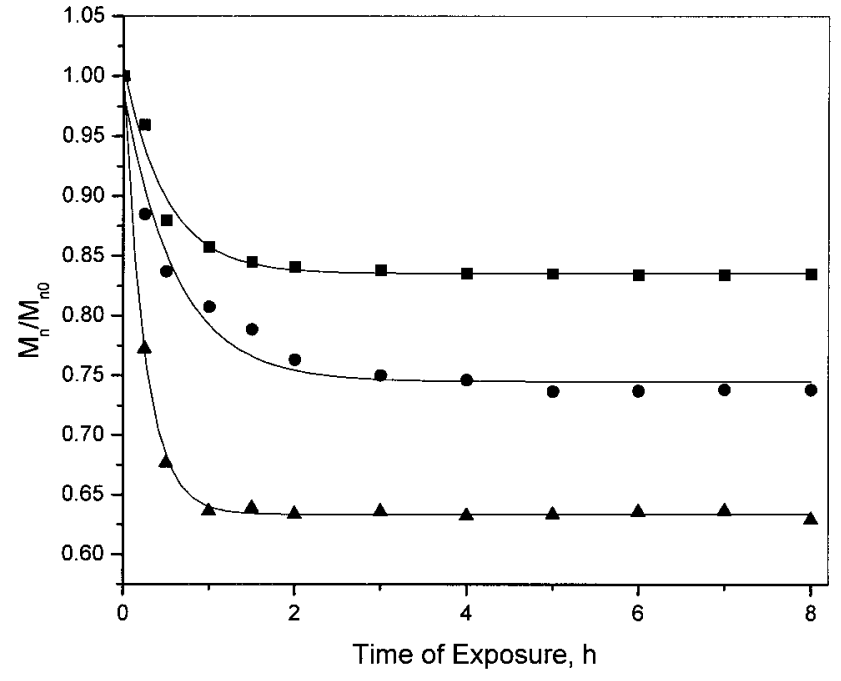

(b)

Figure 3. Variation of the number-average molecular weight with time for the ultrasonic degradation in various solvents.

$\boldsymbol{\square}$, Benzene; $\boldsymbol{\bullet}$, monochlorobenzene $(\mathrm{MCB}) ; \boldsymbol{\Delta}$, dichlorobenzene (DCB); - , model fits. (a) Poly(bisphenol A carbonate); (b) poly( $\varepsilon$-caprolactone); (c) poly(vinyl acetate).

that $M_{n}$ based on moments defined by Eq. 7 decreases continuously until it reaches a value close to 0 . It is also found that the $M_{n}$ value is independent of the parameter $\phi$ and simulations fit the same trend for all the values of this parameter. This can also be observed from the moment

equations (Madras and McCoy, 2001; McCoy and Madras, 1998), which clearly show that the zeroth and first moments are independent of the stoichiometric kernel. This indicates that the variation of the number-average molecular weight is independent of the stoichiometric kernel when $\zeta_{\text {lim }}=0$. The

Table 1. Parameters Used in the Model for the Ultrasonic Degradation of Various Polymers and in Different Solvents*

\begin{tabular}{|c|c|c|c|c|c|c|c|}
\hline Polymer & $M_{n 0}$ & $\mathrm{PD}_{0}$ & Solvent & $\zeta_{\lim }$ & $\left(\mathrm{PD} / \mathrm{PD}_{0}\right)_{\lim }$ & $\phi$ & $\begin{array}{c}k_{d 0} \times 10^{3} \\
\left(\mathrm{~s}^{-1}\right)\end{array}$ \\
\hline \multirow[t]{3}{*}{ PCL } & 80,000 & 1.30 & Benzene & 0.84 & 1.03 & 0.085 & 1.74 \\
\hline & & & MCB & 0.74 & 1.04 & 0.088 & 2.62 \\
\hline & & & DCB & 0.63 & 1.06 & 0.095 & 2.95 \\
\hline \multirow[t]{3}{*}{ PC } & 44,000 & 1.40 & Benzene & 0.77 & 1.03 & 0.086 & 1.90 \\
\hline & & & MCB & 0.59 & 1.05 & 0.091 & 2.64 \\
\hline & & & $\mathrm{DCB}$ & 0.49 & 1.08 & 0.101 & 2.78 \\
\hline \multirow[t]{3}{*}{ PVAC } & 226,000 & 1.32 & Benzene & 0.32 & 1.04 & 0.090 & 2.08 \\
\hline & & & MCB & 0.26 & 1.06 & 0.096 & 3.04 \\
\hline & & & DCB & 0.22 & 1.09 & 0.102 & 3.21 \\
\hline
\end{tabular}

$* M_{n 0}$, average molecular weight; $\mathrm{PD}$, polydispersity. 


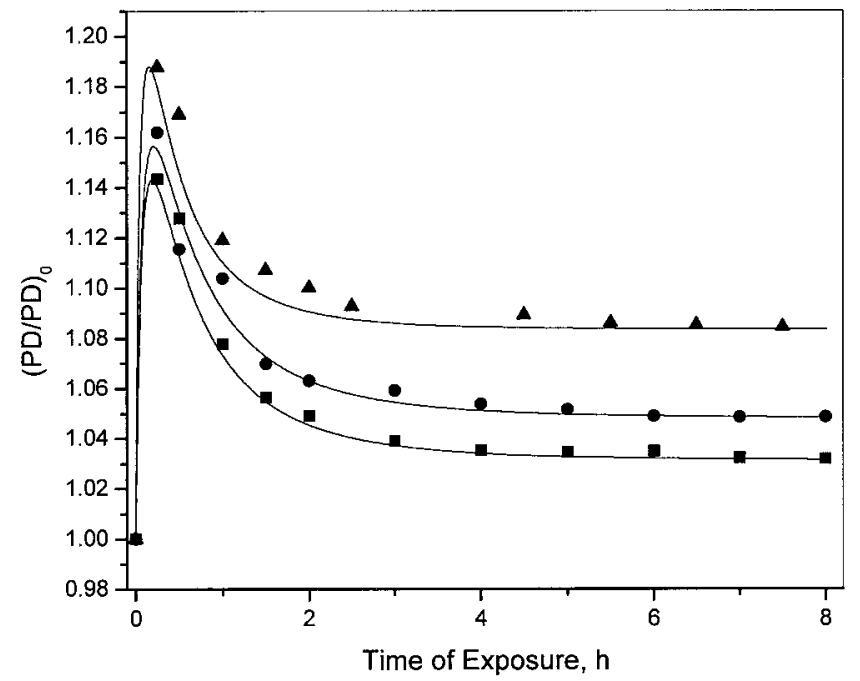

(a)

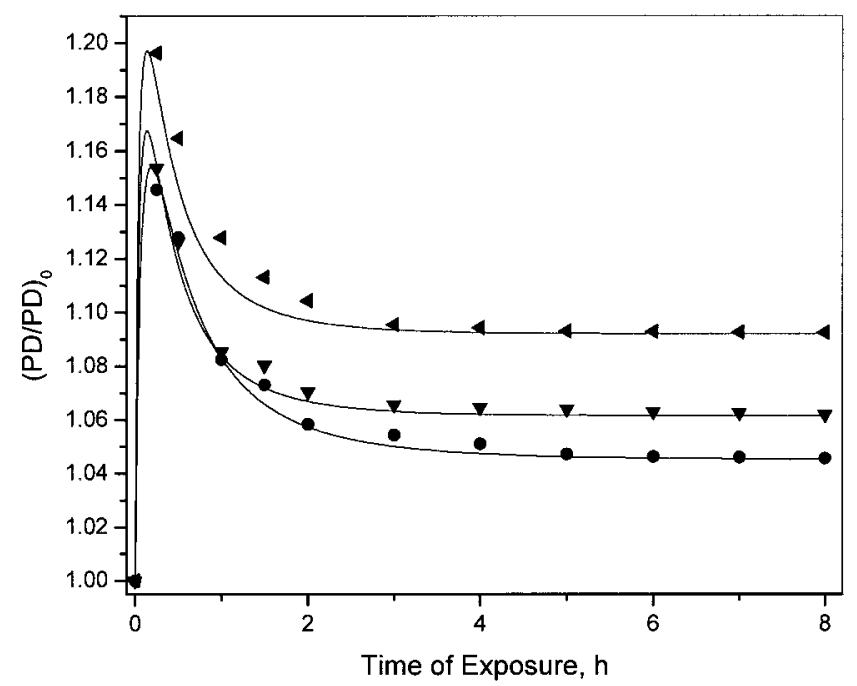

(c)

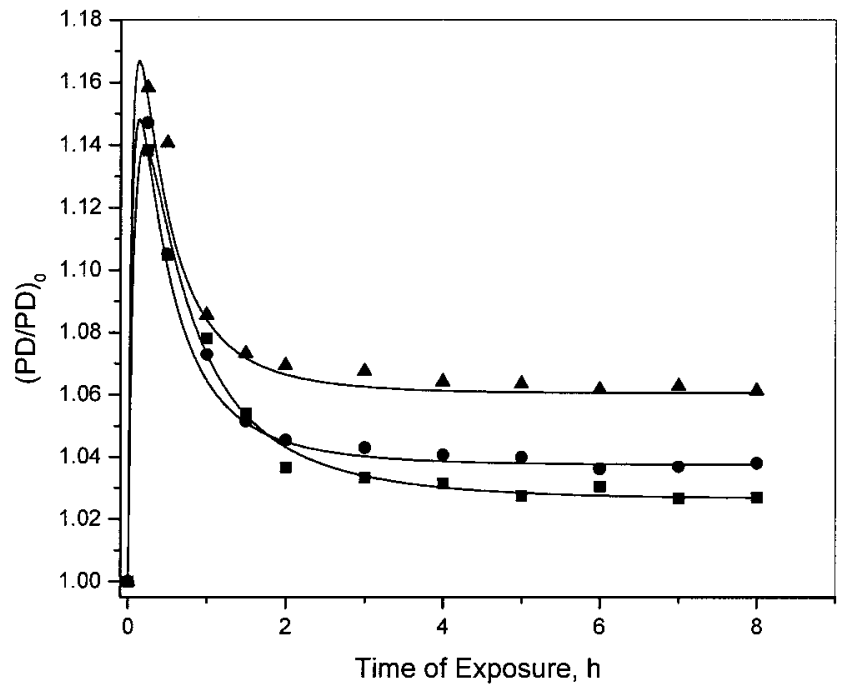

(b)

Figure 4. Time evolution of polydispersity and model fit with modified definition of moments with the parameters listed in Table 1. See Figure 2 for legends.

(a) Poly(bisphenol A carbonate); (b) poly( $\varepsilon$-caprolactone); (c) poly(vinyl acetate).

stoichiometric kernel, however, influences the time evolution of polydispersity.

For low values of $\phi(=0.01)$, where the Lorentzian function closely represents the midpoint chain scission behavior, the trend closely follows that of a Dirac delta function (Madras and McCoy, 2001; McCoy and Madras, 1998). The polydispersity increases monotonically and reaches a constant value well above the initial polydispersity. Higher values of $\phi$ cause the Lorentzian function to spread and would represent a random kernel. For higher values of $\phi(=100)$, the trend approximates that of a purely random chain scission with polydispersity approaching 2, consistent with the observations of McCoy and Madras (1998).

Simulations for intermediate values of $\phi$ are conducted and shown in Figure 2b, which are in between the random and midpoint chain scission. No maximum in the polydispersity is observed, which implies that the mode of scission could not explain the maximum in the polydispersity observed in the experiments. Figure $2 \mathrm{~b}$ shows the polydispersity variation for

the limiting molecular weight case of $\zeta_{\text {lim }}=0.5$, which clearly implies that the maximum polydispersity is attributed to the limiting molecular weight. The polydispersity increases to a maximum and reaches a constant value. The polydispersity always reaches the limiting value of unity and is consistent with values reported in the literature (Madras and McCoy, 2001). The limiting value of unity in the polydispersity is attributed to the moment definition of Eq. 7. At longer times, only polymers with MW values higher than the limiting molecular weight are taken into the domain of the calculation for polydispersity and the polymers of lower molecular weight less than limiting molecular weight is not considered.

For the ideal case of midpoint chain scission, the final polydispersity will be equal to the initial polydispersity. Such limiting cases of the model with Dirac delta functions have been investigated (Madras and McCoy, 2001; McCoy and Madras, 1997, 1998) and the value for the ratio of polydispersity of polymer mixture at any time to the initial polydispersity reduces to unity at longer time $t \rightarrow \infty$ when the moment is 
defined by Eq. 8. If the moment is taken for the entire range of molecular weight $[0, \infty)$, the polydispersity of the polymer after degradation will be higher than the initial polydispersity of the polymer.

The simulations for the various values of $\phi$ with the moment definition of Eq. 8 for the limiting case of $\zeta_{\text {lim }}=0.5$ are presented in Figure 2c, which clearly depicts that the position of the peak and the constant value in the polydispersity are strong functions of the distribution in the breakage around the midpoint. Even for smaller values of $\phi$, the polydispersity offsets from the value of unity, indicating that the offset in saturation in the polydispersity during the ultrasonic degradation is attributed to the distribution in the breakage around the midpoint. It can be clearly seen from the plots that breakage distribution around the midpoint can mimic the experimental observations, as explained below.

\section{Experimental observations}

The ultrasonic degradation of poly(bisphenol A carbonate) [PC], poly( $\varepsilon$-caprolactone) [PCL], and poly(vinyl acetate) [PVAC] was investigated with various solvents such as benzene, monochlorobenzene, and dichlorobenzene. Figures $3 \mathrm{a}-\mathrm{c}$ show the molecular weight variation of PC, PCL, and PVAC, respectively, in various solvents. The results show that degradation rates are strong functions of solvents. The molecular weight decreases sharply initially until it attains a limiting molecular weight. The limiting molecular weights are different for different solvents. The existence of limiting molecular weight and a sharp decrease in the molecular weight, observed by many investigators, are characteristic of ultrasonic degradation (Madras and Chattopadhyay, 2001a,b; Madras et al., 2000; Price, 1990; Price and Smith, 1993a). The solid lines are the fits by the model with moments defined by Eq. 8 and the parameters of the model are given in Table 1.

Figures $4 a-4 c$ show the time variation of polydispersity for the degradation of PC, PCL, and PVAC, respectively. The polydispersity achieves a maximum before attaining a constant value. The maximum in the polydispersity is shown to be attributed to limiting molecular weight, which is a characteristic of ultrasonic degradation (Madras and McCoy, 2001; Madras et al., 2000).

For an ideal midpoint chain scission, the polydispersity attains unity if the moments are defined by Eq. 7 (Madras and McCoy, 2001), whereas the relative polydispersity (ratio of polydispersity of the samples at any instantaneous time to the initial polydispersity) attains unity for the moment defined by Eq. 8 (Madras et al., 2000). Experimental data show that the relative polydispersity attained a constant value greater than unity, indicating that the mode of polymer scission is not ideal midpoint chain scission.

The solid lines in the figures are model fits to the experimental data, with the moment defined by Eq. 8. The model parameters and experimental details are given in Table 1. The plots indicate that the model could fit well over the entire range and the spread parameter of breakage $(\phi)$ lies in the range of 0.08 to 0.11 , indicating a distribution in the breakage around the midpoint of the chain. Thus, the present model, based on the hypothesis of distributed midpoint chain scission, could explain the ultrasonic degradation of polymers. Figure 5 shows the variation of degradation rate coefficient with vapor pressure

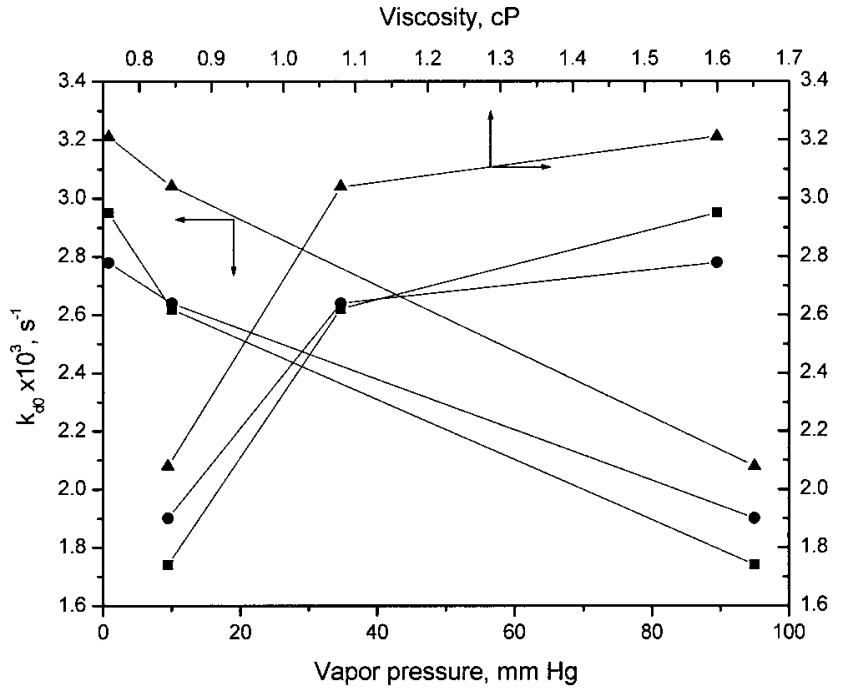

Figure 5. Variation of rate coefficient $k_{d 0}$ with vapor pressure and viscosity.

$\mathbf{\square}, \operatorname{Poly}(\varepsilon$-caprolactone); $\boldsymbol{0}$ poly(bisphenol A carbonate); $\mathbf{\Delta}$, poly(vinyl acetate).

and viscosity for all the polymers. The experimental data indicate that the degradation rate decreases with increase in vapor pressure. Higher vapor pressures cause a cushioning effect on the cavitation bubbles, thereby lessening the shock waves generated (Price, 1990; Price and Smith, 1993a), leading to a reduction of the degradation of the polymer. The increase in viscosity of the solution increased the degradation rates of polymers (Nguyen, 1997) and can be attributed to the hydrodynamic shear forces in the neighborhood of cavitation bubbles with higher cavitation bubble collapse in more viscous solution than in a less viscous solution (Gronroos et al., 2004) and a better transmission of the shock waves in solution of higher kinematic viscosities (Madras and Chattopadhyay, 2001a). The observance of a higher degradation rate at lower vapor pressures and higher kinematic viscosities of the solution is consistent with the experimental data reported in the literature (Chattopadhyay and Madras, 2003; Madras and Karmore, 2001; Price, 1990; Price and Smith, 1993b).

\section{Conclusions}

The ultrasonic degradation of various polymers such as poly(bisphenol A carbonate), poly( $\varepsilon$-caprolactone), and poly(vinyl acetate) was investigated in various solvents such as benzene, monochlorobenzene, and dichlorobenzene. The degradation rates and the limiting molecular weight increased with decrease in the vapor pressure of the solvent and increased with increase in viscosity. The polydispersity showed a maximum during degradation and reached a constant value higher than the initial polydispersity. A continuous distribution model with a stoichiometric kernel based on Lorentzian distribution was developed and used to model the data. The model fits the data satisfactorily and suggests that the mode of scission in the ultrasonic degradation is midpoint with a distribution around it.

\section{Acknowledgments}

The authors gratefully acknowledge the financial support provided by the Department of Science and Technology, India. The first author thanks 
the General Electric Company, USA for a fellowship during his doctoral study.

\section{Literature Cited}

Aris, R., and G. R. Gavalas, "On the Theory of Reactions in Continuous Mixtures," Philos. Trans. R. Soc. London, A260, 351 (1966).

Bak, A. B., and K. Bak, "The Viscosity of Degrading Polymer Solutions," Acta Chem. Scand., 13, 1997 (1959).

Chattopadhyay, S., and G. Madras, "Influence of Temperature on the Ultrasonic Degradation of Poly(vinyl acetate) and Poly(vinyl chloride)," J. Appl. Polym. Sci., 88, 2818 (2003).

Glynn, P. A. R., B. M. E. van der Hoff, and P. M. Reilly, "General Model for Prediction of Molecular Weight Distributions of Degraded Polymers," J. Macromol. Sci. Chem., A6, 1653 (1972).

Grönroos, A., P. Pirkonen, and O. Ruppert, "Ultrasonic Depolymerization of Aqueous Carboxymethylcellulose," Ultrason. Sonochem., 11, 9 (2004).

Hill, P. J., and K. M. Ng, "New Discretization Procedure for the Breakage Equations," 41, 1204 (1995).

Jellinek, H. H. G., ed., Degradation of Vinyl Polymers, Academic Press, New York (1955).

Jellinek, H. H. G., and G. White, "The Degradation of Long Chain Molecules by Ultrasonic Waves: I. Theoretical," J. Polym. Sci., 6, 745 (1951).

Koda, S., H. Mori, K. Matsumoto, and H. Nomura, "Ultrasonic Degradation of Water Soluble Polymers," Polymer, 34, 30 (1993).

Madras, G., and S. Chattopadhyay, "Effect of Solvent on the Ultrasonic Degradation of Poly(vinyl acetate)," Polym. Degrad. Stab., 71, 273 (2001a).

Madras, G., and S. Chattopadhyay, "Effect of Benzoyl Peroxide on the Ultrasonic Degradation of Poly(vinyl acetate)," Polym. Degrad. Stab., 73, 33 (2001b).

Madras, G., and V. Karmore, "Continuous Distribution Kinetics for Ultrasonic Degradation of Poly(methyl methacrylate)," Polym. Int., 50, 683 (2001).

Madras, G., S. Kumar, and S. Chattopadhyay, "Continuous Distribution Kinetics for Ultrasonic Degradation of Polymers," Polym. Degrad. Stab., 69, 73 (2000).

Madras, G., and B. J. McCoy, "Molecular Weight Distribution Kinetics for Ultrasonic Reactions of Polymers," AIChE J., 47, 2341 (2001).

Mason, T. J., and D. Peters, Practical Sonochemistry. Power Ultrasound Uses and Applications, 2nd Edition, Horwood Publishing, Chichester, UK (2002).
McCoy, B. J., and G. Madras, "Degradation Kinetics of Polymers in Solution: Dynamics of Molecular Weight Distributions," AIChE J., 43, 802 (1997).

McCoy, B. J., and G. Madras, "Evolution to Similarity Solutions to Fragmentation and Aggregation," J. Colloid Interface Sci., 210, 200 (1998).

McCoy, B. J., and M. Wang, "Continuous Mixture Fragmentation Kinetics: Particle Size Cracking and Molecular Cracking," Chem. Eng. Sci., 49, 3773 (1994).

Mostafa, M. A., "Degradation of Addition Polymers by Ultrasonic Waves," J. Polym. Sci., 22, 535 (1956).

Nguyen, T. Q., Q. Z. Liang, and H. H. Kausch, "Kinetics of Ultrasonic and Transient Elongational Flow Degradation: A Comparative Study," Polymer, 38, 3783 (1997).

Ovenall, D. W., G. W. Hastings, and D. E. M. Allen, "The Degradation of Polymer Molecules in Solution Under the Influence of Ultrasonic Waves," J. Polym. Sci., 33, 207 (1958).

Price, G. J., "The Use of Ultrasound for the Controlled Degradation of Polymer Solutions," Advances in Sonochemistry, Vol I, T. J. Mason, ed., Cambridge JAI Press, Greenwich, CT, p. 231 (1990).

Price, G. J., Current Trends in Sonochemistry, G. J. Price ed., R.S.C. Special Publication 116, R.S.C., Cambridge, UK, p. 87 (1992).

Price, G. J., D. J. Norris, and P. J. West, "Polymerization of Methyl Methacrylate Initiated by Ultrasound," Macromolecules, 25, 6447 (1992).

Price, G. J., and P. F. Smith, "Ultrasonic Degradation of Polymer in Solutions: 2. The Effect of Temperature, Ultrasound Intensity and Dissolved Gases on Polystyrene in Toluene," Polymer, 34, 4111 (1993a).

Price, G. J., and P. F. Smith, "Ultrasonic Degradation of Polymer in Solutions: 3. Effect of Changing Solvent and Solution Concentration," Polym. J. 29, 419 (1993b).

Price, G. J., P. J. West, and P. F. Smith. "Control of Polymer Structure Using Power Ultrasound,” Ultrason. Sonochem., 1, 51 (1994).

Schmid, G., "Kinetics of Depolymerization by Ultrasonic Waves," Z. Phys. Chem., A186, 113 (1940).

Sivalingam, G., and G. Madras, "Thermal Degradation of Poly( $\epsilon$-caprolactone)," Polym. Degrad. Stab., 80, 11 (2003).

van der Hoff, B. M. E., and P. A. R. Glynn, "Rate of Degradation by Ultrasonication of Polystyrene in Solution," J. Macromol. Sci. Chem., A8, 429 (1974).

Ziff, R. M., "New Solutions to the Fragmentation Equation," J. Phys. A: Math. Gen., 24, 2821 (1991).

Manuscript received Sep. 8, 2003, and revision received Dec. 16, 2003. 